\title{
DISPERSION AND ATTENUATION FOR AN ACOUSTIC WAVE EQUATION CONSISTENT WITH VISCOELASTICITY
}

\author{
A. HANYGA \\ ul. Bitwy Warszawskiej 14 m. 52, 02-366 Warszawa, Poland \\ ajhbergen@yahoo.com
}

\begin{abstract}
An acoustic wave equation for pressure accounting for viscoelastic attenuation is derived from viscoelastic equations of motion. It is assumed that the relaxation moduli are completely monotonic. The acoustic equation differs significantly from the equations proposed by Szabo (1994) and in several other papers. Integral representations of dispersion and attenuation are derived. General properties and asymptotic behavior of attenuation and dispersion in the low and high frequency range are studied. The results are compatible with experiments. The relation between the asymptotic properties of attenuation and wavefront singularities is examined. The theory is applied to some classes of viscoelastic models and to the quasi-linear attenuation reported in seismology.
\end{abstract}

keywords: wave propagation; viscoelasticity; attenuation; completely monotonic functions; complete Bernstein functions; ultrasound

\section{Notation.}

$\begin{array}{lll}f * g & \text { convolution } & \int_{0}^{t} f(s) g(t-s) \mathrm{d} s \\ \mathcal{L}(f)=\tilde{f} & \text { Laplace transform of } f & \int_{0}^{\infty} \mathrm{e}^{-p t} f(t) \mathrm{d} t \\ ] a, b] & \text { the set } a<x \leq b & \\ f(x) \sim_{a} g(x) & \text { asymptotic equivalence } & 0<\lim _{x \rightarrow a} f(x) / g(x)<\infty \text { for } a=0 \text { or } \infty\end{array}$

\section{Introduction.}

Correct modeling of wave attenuation is an important objective for several branches of acoustics and a vast literature is dedicated to this subject. We shall be concerned here with modeling of intrinsic attenuation (i.e. excluding attenuation due to backscatter) in the context of pressure wave equations used in acoustics. In this context many attenuation models have been constructed ad 
hoc to match some aspect of the experimental data without taking into account the physical nature of the wave motion involved.

In many applications the mathematical model of propagation of acoustic pulses (longitudinal waves) in bio-tissues and polymers is based on a linear wave equation for the pressure field. The pressure wave equation can be derived from the equations of motion of linear elasticity. In order to account for attenuation some authors included in the wave equation an additional term involving a fractional time derivative or a more general pseudo-differential operator acting on the time variable [1, 2, 3. This method of accounting for attenuation is called the time causal method in [4]. In this context causality means that the pseudo-differential operator is the convolution with a causal distribution, which ensures the validity of Kramers-Kronig relations. In [5, 6] the time derivatives have been modified in order to ensure that the attenuation obeys a fixed power law with an exponent $>1$ in the entire frequency range. It will be shown that in all these cases the resulting pressure wave equations are inconsistent with the viscoelastic equations of motion.

Introducing attenuation by modifying the time derivatives in the equations of motion is justified in the context of poroelasticity [7, 8, 9, 10, 11, 12, 13. In poroelasticity and poroacoustics the attenuation due to viscous flow in a porous medium is represented by the viscodynamic operator, which is a pseudodifferential operator acting on the time variables [14, 15, 16. In poroelastic equations of motion the viscodynamic operator is applied to the inertial terms. In viscoelasticity attenuation is introduced through a time convolution operator in the constitutive equations. It will be shown in Sec. 2 that in both the viscoelastic equations of motion and in the acoustic wave equation attenuation is represented by a term involving a pseudodifferential operator acting on both the time and spatial variables. This observation is not new, for example Stokes' equation has this structure.

The attempts to explain the experimentally observed power law frequency dependence of attenuation in polymers, bio-tissues and some viscous fluids in terms of an oversimplified power law attenuation model [1, 5, 6] result in unbounded phase speeds. Experiments covering the range 1-250 $\mathrm{MHz}$ indicate that the exponent of the power law lies between 1 and 2. In the oversimplified model it is assumed that the power law extends to the high frequency range. A power law with an exponent $>1$ in the high-frequency range entails wave propagation with unbounded phase speed. It has been shown in [17] that experimental data lie in the low frequency range represented by the low-frequency asymptotics of viscoelastic relaxation models. It is shown in [17 that viscoelastic solids (i.e. viscoelastic media in which strain under constant load does not relax to 0) have power law exponents in the range 1-2 in the low-frequency range. Exponents below one indicate that the material is a viscoelastic fluid such as some bio-tissues subject to a constant shear load These general results are is corroborated by the analysis of specific relaxation models in [18 for longitudinal waves. This indicates that experimental data can be explained in terms of viscoelastic models.

Kowar, Schertzer and coworkers [19, 20] constructed lossy wave equations 
with a bounded propagation speed by applying causal attenuation operators to the elastic pressure wave equation, which ensures bounded phase speed. They found that superlinear attenuation rates exist only in the low frequency range covered by the experiments. In their model, attenuation exhibits sublinear frequency dependence in the high frequency range. This approach however amounts to a modification of the time derivatives in the pressure wave equation, which is not consistent with viscoelastic equations of motion.

If the pressure wave equation is derived from viscoelastic equations of motion but the attenuation grows at a superlinear rate in the high frequency range then the creep compliance is not concave 21. This contradicted by observations. In the special case of strict power law attenuation with an exponent $>1$ in the entire frequency range it is easy to prove that the creep compliance is decreasing and convex.

Several researchers have avoided the problem by working directly with the equations of motion of linear viscoelasticity or thermo-viscoelasticity with an appropriate stress relaxation model [22, 23, 24]. We shall however present an approximate derivation of a linear pressure wave equation based on the equations of motion of viscoelasticity. It will be thus demonstrated that viscoelastic attenuation is represented in the pressure wave equation by a mixed temporal and spatial derivative or a time convolution operator acting on a Laplacian of the pressure field (Sec. 3.2). It will then be shown in Sec. 3 that the attenuation and dispersion associated with the wave equation has the same properties as the attenuation and dispersion in linear viscoelasticity examined in [25, 17]. The theory will be applied to some classes of viscoelastic media.

In Sec. 3.5 we shall establish the relations between the asymptotics of the attenuation function and regularity properties of Green's function at the wavefronts. So far regularity at the wavefronts has only been studied in connection with the singularity of the relaxation modulus or its derivative.

The dispersion-attenuation theory will also be used to examine the controversial linear frequency dependence of attenuation observed in geological media [26, 27, 28. Linear attenuation is incompatible with viscoelasticity and with bounded phase speed. We shall therefore look for the closest approximation to linear attenuation compatible with bounded phase speed. We investigate here attenuation models which differ from linear attenuation by a slowly varying factor.

In Sec. 2 a wave equation for pressure in a viscoelastic medium will be derived. In Sec. 3.2 the dispersion and attenuation theory will be developed for this equation. The dissipation-attenuation theory presented in Sec. 3 depends on the assumption that the viscoelastic relaxation modulus is a completely monotonic (CM) function [29]. The CM property is so universal in viscoelasticity that several attempts have been made to justify it by an a priori argument [30, 31, 32. In particular every spring-dashpot model and every fractional generalization of such models has a completely monotonic relaxation modulus. The last statement is an easy consequence of the duality theorem proved in 29. Many other models of viscoelastic relaxation (Prony series, the Havriliak-Negami model and its special cases) are also expressed in terms CM relaxation moduli. 
In Sec. 3.5 it is shown that the order of the wavefront singularity in media with bounded wavefront speed depends on the asymptotic behavior of the attenuation function at infinity.

In Sec. 4 the general theory is applied to some examples involving strongly singular convolution operators ( $K$ is singular at 0 but locally integrable, the phase speed is unbounded, Sec. 4.3), weakly singular kernels ( $K$ is non-singular, but its derivative $K^{\prime}$ has a singularity at 0 , Sec. 4.2 and non-singular kernels ( $K$ and $K^{\prime}$ are continuous at 0 , the attenuation function is bounded). In the first case the phase speed is unbounded while in the second case the wave fields exhibit finite wavefront speed. In Section 4.4 the case of nearly linear frequency dependence of attenuation is examined.

\section{Derivation of the pressure wave equation.}

Consider a homogeneous isotropic compressible viscous fluid defined by the constitutive equation

$$
\begin{gathered}
\sigma=-P \mathbf{I}+\lambda \operatorname{div} \mathbf{u}_{, t} \mathbf{I}+\mu\left[\nabla \mathbf{u}_{, t}+\left(\nabla \mathbf{u}_{, t}\right)^{\top}\right] \\
P=c_{0}^{2}\left(\rho-\rho_{0}\right)
\end{gathered}
$$

where $\sigma$ denotes the Cauchy stress tensor, $P$ represents the elastic part of the pressure, $\mathbf{u}$ is the displacement vector, $\rho$ is the density and $\rho_{0}$ is a reference density. The parameters $K:=\lambda+2 \mu$ and $\mu$ are dynamic viscosities of volumetric and shear deformations and $c_{0}$ is an elastic propagation speed.

Mass conservation can be expressed in the form $\tau_{, t}=\tau \operatorname{div} \mathbf{u}_{, t}$, where $\tau:=$ $1 / \rho$. Hence

$$
\rho_{, t}=-\rho \operatorname{div} \mathbf{u}_{, t}
$$

For further reference we note that $\rho-\rho_{0}=\rho_{0}[\exp (-\operatorname{div} \mathbf{u})-1] \approx-\rho_{0} \operatorname{div} \mathbf{u}$ and thus equation (2) assumes the form $P \approx-\mathcal{K} \operatorname{div} \mathbf{u}, \mathcal{K}=\rho_{0} c_{0}^{2}$, in the linear approximation.

Equation (3) implies that

$$
\operatorname{div} \sigma=-\nabla P+(\lambda+\mu) \nabla \operatorname{div} \mathbf{u}_{, t}+\mu \nabla^{2} \mathbf{u}_{, t}
$$

The momentum balance $\rho \mathbf{u}_{, t t}=\operatorname{div} \sigma$ and the mass balance imply the following equation for $\xi:=\operatorname{div} \mathbf{u}$ :

$$
\rho \xi_{, t t}=-\nabla^{2} P+(\lambda+2 \mu) \nabla^{2} \xi_{, t}
$$

Eqs (2) and (3) imply that $c_{0}^{-2} P_{, t}=-\rho \xi_{, t}$ and $c_{0}^{-2} P_{, t t}=-\rho \xi_{, t t}-\rho_{, t} \xi_{, t}=$ $-\rho\left(\xi_{, t t}-\xi_{, t}^{2}\right)$. In a linearized theory we assume that

$$
\left|\xi_{, t}\right|^{2} \ll\left|\xi_{, t t}\right|
$$

hence $c_{0}^{-2} P_{, t t}=-\rho \xi_{, t t}$. The final pressure equation is obtained by substituting the last results in (4)

$$
c_{0}^{-2} P_{, t t}=\nabla^{2} P+\frac{\lambda+2 \mu}{\rho_{0} c_{0}^{2}} \nabla^{2} P_{, t}
$$


If multiplication by $\lambda$ and $\mu$ in eq. (1) is replaced by convolutions with CM kernels $\lambda(t)$ and $\mu(t)$, respectively, then eq. (6) assumes the following form

$$
c_{0}^{-2} P_{, t t}=\nabla^{2} P+\left(\rho_{0} c_{0}^{2}\right)^{-1} \nabla^{2} K * P_{, t}
$$

for $x \in \mathbb{R}^{d}, d=1$ or 3 . The convolution kernel $K(t):=\lambda(t)+2 \mu(t)$ is a $\mathrm{CM}$ function.

We shall consider the Cauchy problem for equation (7) with the initial conditions

$$
\begin{gathered}
P(t, x)=0 \quad \text { for } t<0, \\
\lim _{t \rightarrow 0+} P(t, x)=P_{0}(x), \quad \lim _{t \rightarrow 0+} P_{, t}(t, x)=Q_{0}(x)
\end{gathered}
$$

\section{Dispersion and attenuation theory.}

\subsection{Mathematical preliminaries.}

We shall recall the notion of completely monotonic functions and complete Bernstein functions $(\mathrm{CBF})$ and some properties of the latter class of functions. For more details see [33] or [17].

Definition 1 A real function $f$ defined on $] 0, \infty[$ is said to be completely monotonic (CM) if it is infinitely differentiable and its derivatives satisfy the inequalities

$$
(-1)^{n} \mathrm{D}^{n} f(t) \geq 0
$$

for $n=0,1,2, \ldots$

A CM function can have a singularity at 0 . Any linear combination of $\mathrm{CM}$ functions with positive coefficients is obviously CM.

If $f$ is $\mathrm{CM}$ and it is integrable over $[0,1]$, then $f$ is said to be locally integrable CM (LICM).

According to Bernstein's theorem [34] a CM function can be expressed in terms of a positive Radon measure:

Theorem 1 If $f$ is a CM function then there is a positive Radon measure $m$ such that

$$
f(x)=\int_{[0, \infty[} \mathrm{e}^{-x y} m(\mathrm{~d} y), \quad x>0
$$

For our purposes a Radon measure is essentially a measure with infinite mass. A CM function $f$ is locally integrable if and only if the measure $m$ satisfies the inequality

$$
\int_{[0, \infty[} \frac{m(\mathrm{~d} y)}{1+y}<\infty
$$

A function $f$ on $] 0, \infty[$ is said to be a Bernstein function (BF) if it is differentiable and its derivative is LICM. A BF is thus non-negative and non-decreasing, hence it has a finite limit at 0 . 
Definition $2 A$ real function $f$ on $\overline{\mathbb{R}_{+}}$is a $C B F$ if and only if there is a $B F g$ such that $f(x)=x^{2} \tilde{g}(x)$.

If $f$ is locally integrable $\mathrm{CM}$, then $p \tilde{f}(p)$ is a CBF, where $\tilde{f}$ denotes the Laplace transform of $f$.

The theorems on CBFs cited below can be found in the monograph 33 .

Theorem 2 Every CBF $f$ has an integral representation

$$
f(x)=a+b x+x \int_{] 0, \infty[} \frac{\nu(\mathrm{d} r)}{x+r}, \quad x \geq 0
$$

with $a, b \geq 0$ and a positive Radon measure $\nu$ satisfying the inequality

$$
\int_{] 0, \infty[} \frac{\nu(\mathrm{d} r)}{1+r}<\infty
$$

The integral in equation (11) is a decreasing function of $x$. Consequently

$$
\lim _{x \rightarrow \infty} f(x) / x=b
$$

while

$$
\lim _{x \rightarrow 0} f(x)=a
$$

Theorem $3 A$ non-zero function $f$ is a $C B F$ if and only if the function $x / f(x)$ is a $C B F$.

Theorem 4 If $f$ is a $C B F$ and $0 \leq \alpha \leq 1$ then $f(x)^{\alpha}$ is also a $C B F$.

Definition 3 A real function $f$ defined on $[0, \infty[$ is slowly varying at $w=0$ or $\infty$ if for all $\lambda>0$

$$
\lim _{x \rightarrow w} f(\lambda x) / f(x)=1 .
$$

The logarithm $\ln (1+x)$ has this property.

Definition 4 A real function $f$ defined on $[0, \infty[$ is regularly varying at at $w=0$ or $\infty$ if for all $\lambda>0$ the limit $\lim _{x \rightarrow w} f(\lambda x) / f(x)$ is finite.

If $f$ is regularly varying at $w$ then $f(x) \sim_{w} x^{\alpha} l(x)$ for some real $\alpha$ and a function $l(x)$ slowly varying at $w$, where $w$ is either 0 or infinity.

Theorem 5 (Valiron 1911)

If $f$ is an increasing function satisfying the condition $\lim _{y \rightarrow-0} f(y)=0$ and $g(x)$ is given by the Stieltjes integral

$$
g(x)=\int_{[0, \infty[} \frac{\mathrm{d} f(y)}{x+y}
$$

$0 \leq \beta<1$ and the function $l$ is slowly varying at infinity, then the following two statements are equivalent: 
(i) $f(y) \sim y^{\beta} l(y)$ for $y \rightarrow \infty$;

(ii) $g(x) \sim[(\pi \beta) / \sin (\pi \beta)] x^{\beta-1} l(x)$.

35.

Theorem 5 can also be applied to integrals of the form $\int_{[0, \infty[} \mu(\mathrm{d} y) /(x+y)$ by setting $f(y)=\mu([0, y])$ for $y \geq 0$ and $f(y)=0$ if $y<0$.

\subsection{Application to the dispersion-attenuation theory.}

The attenuation-dispersion theory will be presented along the lines of [17. Although there are some differences between (15) below and eq. (9) in op. cit., the analysis of attenuation and dispersion is very similar. We shall therefore present the main arguments in brief. Many other results obtained in [17] can be extended to the wave equation under consideration without changing the argument.

The wavenumber (or the length of the wave number vector) of a pressure field satisfying equation (7) is given by the formula $k=\mathrm{i} \kappa(-\mathrm{i} \omega)$, where

$$
\kappa(p)=\frac{p}{c_{0}}\left(1+p \tilde{K}(p) /\left(\rho_{0} c_{0}^{2}\right)\right)^{-1 / 2}
$$

The convolution kernel $K(t)$ is assumed to be a locally integrable completely monotonic function, hence

$$
K(t)=\int_{[0, \infty[} \mathrm{e}^{-r t} \lambda(\mathrm{d} r)
$$

where $\lambda$ is a positive Radon measure satisfying the inequality

$$
\int_{[0, \infty[} \frac{\lambda(\mathrm{d} r)}{1+r}<\infty
$$

Theorem 2 implies that $p \tilde{K}(p)=p \int_{[0, \infty[} \lambda(\mathrm{d} r) /(r+p)$ is a CBF.

By Theorem 3 and Theorem 4 the function $\kappa$ is a CBF. Consequently the theory of attenuation and dispersion developed for linear viscoelastic media applies for the acoustic equation (7).

Since $\kappa(0)=0$, Theorem 2 implies that

$$
\kappa(p)=B p+\beta(p)
$$

where $B \geq 0$,

$$
\beta(p):=p \int_{] 0, \infty[} \frac{\nu(\mathrm{d} r)}{r+p}
$$

and $\nu$ is a positive Radon measure satisfying the inequality

$$
\int_{] 0, \infty[} \frac{\nu(\mathrm{d} r)}{r+1}<\infty
$$


The support of $\nu$ is called the attenuation spectrum. Equation (17) implies that $\beta(p) / p=\mathrm{o}[1]$ for $p \rightarrow \infty$ uniformly in the closed right-half complex plane [17. This in turn implies that the wavefield is bounded by a wavefront moving with the speed $C_{0}=1 / B$ provided $B>0$. If $B=0$ then the phase speed is unbounded and the wavefield immediately spreads to the entire available space. The constant $B$ is given by the limit $B=\lim _{p \rightarrow \infty} \kappa(p) / p$ (see Appendix A). For some functions $K(t)$ the Radon measure $\nu$ can be calculated explicitly using the analytic properties of the function $\kappa(p)$ as given by eq. [15], cf [17].

The function $\beta$ can be split into its real part $\mathcal{A}$ (attenuation) and imaginary part $-\mathcal{D}$ (excess dispersion). These two quantities will be expressed as functions of the circular frequency $\omega=\mathrm{i} p$ on the real $\omega$ axis. We then have

$$
\begin{aligned}
& \mathcal{A}(\omega)=\omega^{2} \int_{] 0, \infty[} \frac{\nu(\mathrm{d} r)}{\omega^{2}+r^{2}} \\
& \mathcal{D}(\omega)=\omega \int_{] 0, \infty[} \frac{r \nu(\mathrm{d} r)}{\omega^{2}+r^{2}}
\end{aligned}
$$

Equations 19 20 express the two functions $\mathcal{A}$ and $\mathcal{D}$ in terms of the same measure $\nu$. They can be viewed as a parametric form of the dispersion relations. It will be seen that the parametric form of the dispersion relations is often more convenient to use than the Kramers-Kronig relations.

It is clear from $(20)$ that the function $\mathcal{D}$ has sublinear growth in the highfrequency range, i. e. $\mathcal{D}(\omega) / \omega \rightarrow 0$ as $\omega \rightarrow \infty$.

Since $y \rightarrow y /\left(r^{2}+y\right)$ is an increasing function for every $r>0$, equation (19) implies that the attenuation function $\mathcal{A}(\omega)$ is increasing unless $\nu=0$. The statement about the asymptotic behavior of $\beta(p) / p$ at infinity made previously implies that the attenuation function $\mathcal{A}(\omega)$ is also sublinear in the high-frequency range.

If $\nu$ has a finite total mass

$$
M:=\int_{] 0, \infty[} \nu(\mathrm{d} r)<\infty
$$

then $\lim _{\omega \rightarrow \infty} \mathcal{A}(\omega)=M[17$.

The phase speed $c(\omega)$ is defined as $\omega / \operatorname{Re} k(\omega)$. The definition of the dispersion function $\mathcal{D}$ implies that

$$
\frac{1}{c(\omega)}=\frac{1}{C_{0}}+\frac{\mathcal{D}(\omega)}{\omega} .
$$

But

$$
\frac{\mathcal{D}(\omega)}{\omega}=\int_{] 0, \infty[} \frac{r \nu(\mathrm{d} r)}{r^{2}+\omega^{2}} \geq 0
$$

hence $c(\omega) \leq C_{0}$. If $K_{0}:=\lim _{t \rightarrow 0} K(t)<\infty$ then $\lim _{p \rightarrow \infty}[p \tilde{K}(p)]=K_{0}$ in the right half-plane $\operatorname{Re} p \geq 0$ and

$$
C_{0}=\lim _{p \rightarrow \infty} \frac{p}{\kappa(p)}=c_{0}\left(1+K_{0} / \mathcal{K}\right) \geq c_{0}
$$


Equation 20 implies that $\mathcal{D}(\omega) / \omega$ is a decreasing function of frequency, hence $c(\omega)$ is an increasing function of $\omega$. Also $\mathcal{D}(\omega) / \omega \rightarrow 0$ for $\omega \rightarrow \infty$, hence $\lim _{\omega \rightarrow \infty} c(\omega)=C_{0}$.

On the other hand

$$
\lim _{p \rightarrow 0} \frac{\kappa(p)}{p}=c_{0}^{-1}\left(1+K_{\infty} / \mathcal{K}\right)^{-1 / 2}
$$

where $K_{\infty}:=\lim _{t \rightarrow \infty} K(t)=\lim _{p \rightarrow 0}[p \tilde{K}(p)] \geq 0$. The limit 22 exists for arbitrary azimuths $\arg p \in[-\pi / 2, \pi / 2]$, hence $\lim _{\omega \rightarrow 0} c(\omega)=C_{\infty}:=c_{0}\left(1+K_{\infty} / \mathcal{K}\right)^{1 / 2}>$ 0 . We also note that in view of equation (21) for $\omega \rightarrow 0$ the ratio $\mathcal{D}(\omega) / \omega$ tends to the finite limit $1 / C_{\infty}-1 / C_{0}$. Equation (20) implies that this limit is equal to $\int_{] 0, \infty[} \nu(\mathrm{d} r) / r$. An important conclusion is that the last integral is finite:

$$
\int_{] 0, \infty[} \frac{\nu(\mathrm{d} r)}{r}<\infty
$$

Since the function $K$ is non-increasing, the inequality $K_{\infty} \leq K_{0}$ holds and thus $C_{\infty} \leq C_{0}$. Hence the phase speed increases monotonically from $C_{\infty}$ to $C_{0}$.

The parameter $K_{\infty}$ can be eliminated without changing the model by subtraction $K_{\infty}$ from $K(t)$ and adding it to $\mathcal{K}: \mathcal{K} \rightarrow \overline{\mathcal{K}}=\mathcal{K}+K_{\infty}, K(t) \rightarrow \bar{K}(t)=$ $K(t)-K_{\infty}$. In terms of $\overline{\mathcal{K}}$ and $\bar{K}(t)$ we have $\bar{K}_{\infty}=0, \bar{C}_{\infty}=c_{0}$.

\subsection{Asymptotic behavior of attenuation.}

If $\nu$ has regular variation at $\infty$ and $\nu([0, r]) \sim_{\infty} a r^{\alpha} l(r)$, where $l(r)$ is a function slowly varying at $\infty$ and $a, \alpha>0$, then in view of the inequality 18$) \alpha<1$. It is shown in [17] that $\mathcal{A}(\omega) \sim L(\omega) \omega^{\alpha}$, where $L$ is slowly varying at $\infty$.

If the Radon measure $\nu$ is regularly varying at $0, \nu([0, r]) \sim_{0} l_{1}(r) r^{\gamma}$, then in view of the inequality (23) the exponent $\gamma$ must be greater than 1 . Theorem 1.11 in [17] implies that $\mathcal{A}(\omega) \sim_{0} \omega^{\gamma} L_{1}(\omega)$, where $L_{1}$ is a slowly varying function at 0 . This result is confirmed by a frequently reported experimental observation for longitudinal waves in polymers and bio-tissues for frequencies in the range 0-250 MHz 3. This observation is often misinterpreted as evidence of a power law behavior of attenuation $\mathcal{A}(\omega)=A \omega^{\gamma}, \gamma>1$ valid for all the frequencies.

In the exceptional case $R:=\int_{] 0, \infty[} \nu(\mathrm{d} r)<\infty$ the inequality $\nu(\mathrm{d} r)<$ $\nu(\mathrm{d} r) /\left(1+r^{2} / \omega^{2}\right)$ implies that $\lim _{\omega \rightarrow \infty} \mathcal{A}(\omega)=R$.

Another frequently reported behavior $\mathcal{A}(\omega) \sim a \omega^{2}$ for $\omega \rightarrow 0$ (cf [36, 37]) occurs when the support of the measure $\nu$ is contained in $[b, \infty[$, where $b>0$. In this case

$$
\int_{] 0, \infty[} \frac{\nu(\mathrm{d} r)}{r^{2}+\omega^{2}} \rightarrow \int_{] b, \infty[} \frac{\nu(\mathrm{d} r)}{r^{2}}=: a<\infty
$$

as $\omega \rightarrow 0$, which implies the quadratic behavior of attenuation at low frequencies. According to equation (15) this behavior is expected in the case of Newtonian viscosity $(K(t)=N \delta(t), p \tilde{K}(p)=N p)$ and, more generally, when $p \tilde{K}(p) \sim a p$ for $p \rightarrow 0$ and a constant $a$. 


\subsection{Kramers-Kronig dispersion relations.}

Equation 16 implies that $\beta(p)$ is the Laplace transform of a causal distribution fı, where

$$
f(s)=\left\{\begin{array}{cl}
\int_{0}^{\infty} \mathrm{e}^{-s r} \nu(\mathrm{d} r), & s>0 \\
0, & s \leq 0
\end{array}\right.
$$

Indeed, in view of the inequality 18 the function $f$ is locally integrable. Hence its primitive $g$ of $f$ is continuous and the function $f$ can be viewed as a distribution of first order. Thus $\beta(p)=p \tilde{f}(p)=\mathcal{L}\left(f^{\prime}\right)(p)$ is the Laplace transform of the causal distribution $f^{\prime}$ of second order. If $\mathcal{A}(\omega) \sim$ const $\times \omega^{\alpha}$ for $\omega \rightarrow \infty$, where $0<\alpha<1$, then $\mathcal{A} /\left(1+\omega^{2}\right)$ and $\mathcal{D}(\omega) /\left(1+\omega^{2}\right)$ are integrable. Since these functions are the real and imaginary part of a Fourier transform of a causal function, they satisfy the Kramers-Kronig dispersion relations with one subtraction (38, Sec. 1.8(f), pp. 42-43).

It is however more convenient to work with the parametric Kramers-Kronig relations 19 because they do not involve singular integrals.

\subsection{Regularity of Green's function at the wavefronts.}

Low-frequency asymptotics of the attenuation function can be verified by experiments. High-frequency asymptotics of $\mathcal{A}(\omega)$ is not accessible to such a verification but it determines the regularity at the wavefronts. The influence of the singularity of the relaxation modulus and its derivative at 0 has been examined in several papers [39, 40, 41, 42, 43, 44, 45, 46. In this section we shall however link regularity at the wavefront to the asymptotic behavior of the attenuation function at infinity.

If $C_{0}=\infty$ then Green's function does not vanish anywhere and is an analytic function of $t$ and $\mathbf{x} 39$. In the remaining cases Green's function vanishes outside the wavefront $|\mathbf{x}|=C_{0} t$ and is an analytic function of $t$ and $\mathbf{x}$ in the region of space-time defined by the inequality $t-|\mathbf{x}| / C_{0}>0$. The transition between this region and the unperturbed region is determined by the discontinuities of Green's function and its derivatives at the wavefront or lack thereof. We shall say that the order of the wavefront singularity is $N$ if if at least one derivative $\partial_{t}{ }^{m} \partial_{x}{ }^{n} P(t, x)$ with $n+m=N$ has a jump discontinuity at the wavefront and this statement is not true for any derivatives with $m+n<N$. Green's function of (7) is given by the solution $(36)$ for $P_{0}(x)=0, Q_{0}(x)=\delta(x)$

$$
P(t, x)=\frac{1}{4 \pi} \int_{-\infty}^{\infty} F_{1}(-\mathrm{i} \omega) \mathrm{e}^{-\mathrm{i} \omega(t-|x| / c(\omega))-\mathcal{A}(\omega) r} \mathrm{~d} \omega
$$

with $F_{1}(p)=\kappa(p) / p$ and $r:=|x|$. Note that $|\kappa(-\mathrm{i} \omega) / \omega|^{2}=c(\omega)^{-2}+\mathcal{A}(\omega)^{2} / \omega^{2}$. The second term is bounded for $\omega>\Omega$, where $\Omega$ is an arbitrary positive number, because $\mathcal{A}(\omega)$ is sublinear. The first term is bounded by $c(\Omega)^{-2}$ because $c(\omega)$ is non-decreasing. Let $W_{0}(\omega, r):=C_{1} \exp (-\mathcal{A}(\omega) r)$. The absolute value of $F_{1}$ is bounded for $\omega>\Omega$ and the absolute value of the integrand is majorized by the function $W_{0}(\omega, r)$, for $\omega>\Omega$, where $C_{1}$ is a positive constant. 
Distributional derivatives of $P(t, x)$ are given by the inverse Fourier transform

$$
\partial_{t}^{m} \partial_{x}^{n} P(t, x)=\frac{1}{4 \pi} \int_{-\infty}^{\infty} F_{1}(-\mathrm{i} \omega)(-\mathrm{i} \omega)^{m}(-\kappa(-\mathrm{i} \omega))^{n} \mathrm{e}^{-\mathrm{i} \omega(t-r / c(\omega))-\mathcal{A}(\omega) r} \mathrm{~d} \omega
$$

The absolute value of the integrand is majorized by a function $W_{m, n}(\omega, r):=$ $C_{2} \omega^{m+n} \exp (-\mathcal{A}(\omega) r)$.

Note that the attenuation function is non-negative and non-decreasing, hence

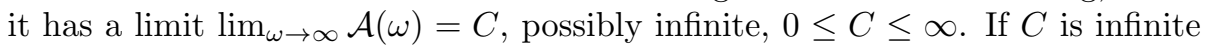
then $\mathcal{A}(\omega)$ can be increasing in the high-frequency range at a logarithmic rate

$$
\mathcal{A}(\omega) \sim_{\infty} A \ln ^{1+\gamma}(\omega), \quad A>0, \gamma>-1
$$

or according to the power law

$$
\mathcal{A}(\omega) \sim_{\infty} A \omega^{\alpha}, \quad 0<\alpha<1
$$

The attenuation is often bounded in the case of a bounded attenuation spectrum, which is the case for the Cole-Davidson relaxation [17] as well as for the Strick and Becker creep [47.

We now assume that the wavefront speed $C_{0}<\infty$.

If the attenuation function satisfies equation (27) then for every positive constant $\varepsilon$ and for $r \geq \varepsilon$ the absolute values of the integrands of (24) and 25) are majorized by the functions $W_{0}(\omega, \varepsilon)$ and $W_{m, n}(\omega, \varepsilon)$ are integrable over $[\Omega, \infty[$ and and consequently $P(t, x)$ and its derivatives of arbitrary order are continuous everywhere outside the origin, in particular at the wavefront if $t>0$. Since they vanish outside the wavefront, they tend to zero at the wavefront. Consequently the signal propagates with a delay with respect to the wavefront and is preceded by a flat "pedestal" [48] (Fig. 1). The importance of the pedestal for seismic inversion was demonstrated in [11].

If $\mathcal{A}(\omega)$ is bounded then the function $W_{0}(\omega, r)$ is not integrable and Green's function $P(t, x)$ can be discontinuous at the wavefront. This effect is demonstrated numerically for the Strick and Becker creep in [49]. This effect is frequent if the attenuation spectrum is bounded, like in the case of relaxation models defined in terms of finite Prony sums, the Cole-Davidson relaxation [17] and the Strick-Becker creep compliance [47]. The appearance of discontinuities at the wavefronts in the last-mentioned case is demonstrated numerically in 49 .

A curious intermediary situation arises if $\mathcal{A}(\omega)$ satisfies equation 26) with $\gamma=0$. In this case $W_{0}(\omega, r) \sim_{\infty} C_{1} \omega^{-A r}$ is integrable if the propagation distance is sufficiently large: $r>1 / A$, while $W_{m, n}(\omega, r) \sim_{\infty} C_{2} \omega^{m+n-A r}$ is integrable if $r>(m+n+1) / A$. At the wavefront $r=c_{0} t$ and thus $P(t, x)$ is continuous at the wavefront for $t>1 /\left(A c_{0}\right)$, while the derivatives of order $N$ are continuous at the wavefront for $t>(N+1) /\left(A c_{0}\right)$. The order of the wavefront singularity thus increases stepwise with time. This effect was deduced by a different argument by Desch and Grimmer [43, 44. 


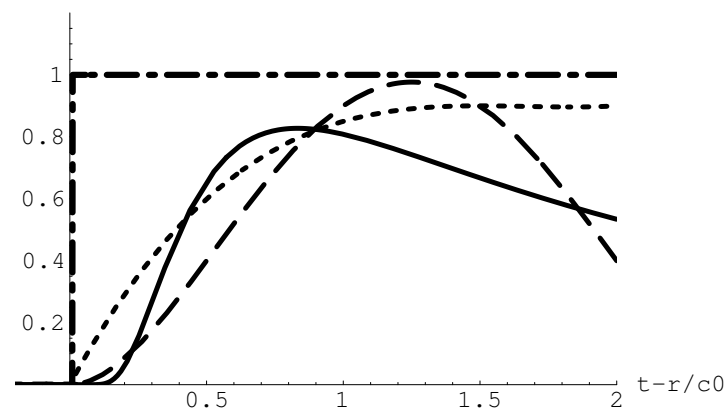

Figure 1: Cross-section of Green's function near the wavefront. (a) Green's function and all its derivatives are continuous at the wavefront (solid line); (b) Green's function and its first-order derivatives are continuous (dashed line); (c) Green's function has a jump discontinuity at the wavefront (dot-dashed line); (d) Green's function is continuous at the wavefront but its derivatives are not (dotted line).

If $\gamma>1$ then $W_{0}(\omega, r)=C_{1} \omega^{-A r \ln ^{\gamma}(\omega)}$ and $W_{m, n}(\omega, r)=C_{2} \omega^{m+n-A r \ln ^{\gamma}(\omega)}$ are integrable for all $r \geq \varepsilon>0$. Consequently $P(t, x)$ and its derivatives are continuous outside the origin, in particular at the wavefront if $t>0$. The regularity properties of Green's function are thus the same as for power law attenuation.

If $-1<\gamma<0$, then $W_{0}(\omega)=C_{1} \omega^{-A r / \ln ^{\gamma}(\omega)}$ and $W_{m, n}(\omega)=C_{2} \omega^{m+n-A r / \ln ^{\gamma}(\omega)}$. In this case both $P(t, x)$ and its derivatives can be discontinuous. It follows that strictly logarithmic growth of attenuation $(\gamma=1)$ constitutes a sharp boundary between media which allow for discontinuity propagation and those which do not allow for discontinuities at the wavefronts.

Equation (37) implies that the order of the wavefront in three-dimensional space is higher by one than in one-dimensional space for the same attenuation function.

\section{Examples.}

\subsection{Viscoelastic media with a bounded attenuation func- tion.}

A special class of viscoelastic relaxation models is characterized by the inequalities $K_{0}<\infty$ and $K_{0}^{\prime}:=K^{\prime}(0)>-\infty$. A frequent representative of this class is a the Dirichlet series $K(t)=\sum_{n=1}^{N} \lambda_{n} \mathrm{e}^{-r_{n} t}$, with $N \leq \infty, \lambda_{n}, r_{n}>0$ for $n=1, \ldots N, \sum_{n=1}^{N} \lambda_{n}=K_{0}<\infty$ and $\sum_{n=1}^{N} r_{n} \lambda_{n}=-K_{0}^{\prime}<\infty$. A finite Dirichlet series is known as the Prony series. This kind of relaxation mechanism is often used to model multiple relaxation due to several relaxation mechanisms such as chemical reactions, cf [22. In seismology it was suggested by Liu et al. 
[50] In this class

$$
\int_{] 0, \infty[} r \lambda(\mathrm{d} r)=-K_{0}^{\prime}<\infty
$$

and therefore

$p \tilde{K}(p)=p \int_{] 0, \infty[} \frac{\lambda(\mathrm{d} r)}{1+r / p} \approx \int_{] 0, \infty[} \lambda(\mathrm{d} r)-\frac{1}{p} \int_{] 0, \infty[} r \lambda(\mathrm{d} r)+\mathrm{o}[1 / p]=K_{0}+K_{0}^{\prime} / p+\mathrm{o}[1 / p]$

for $p \rightarrow \infty$, Re $p \geq 0$, so that

$$
\frac{\kappa(p)}{p} \approx C_{0}^{-1}+\frac{R}{p}
$$

with $R=-K_{0}^{\prime}\left[1+K_{0} / \rho c_{0}^{2}\right]^{-3 / 2} /\left(2 \rho c_{0}^{3}\right)$. Note that

$$
\frac{\kappa(p)}{p}=\frac{p}{C_{0}}+\int_{] 0, \infty[} \frac{\nu(\mathrm{d} r)}{1+r / p} \approx \frac{p}{C_{0}}+R
$$

for large $p$ implies that $\int_{] 0, \infty[} \nu(\mathrm{d} r)=R$. As it has already been noted, this implies that the attenuation function tends to a constant as $\omega \rightarrow \infty$.

\subsection{Viscoelastic media with a asymptotic power law at- tenuation and weakly singular $K(t)$.}

The attenuation function grows at a power law rate in the Cole-Cole relaxation model [51], originally proposed for dielectric relaxation and subsequently applied in polymer viscoelasticity by Bagley and Torvik [52:

$$
p \tilde{K}(p)=M \frac{1+a(\tau p)^{-\alpha}}{1+(\tau p)^{-\alpha}}-M a
$$

with $M, \tau, a>0,0<\alpha<1$. We have subtracted a constant term $M a$ so that $K_{\infty}=\lim _{p \rightarrow 0}[p \tilde{K}(p)]=0$ because a non-zero static modulus is already represented by $\mathcal{K}$. Since $K_{0}=\lim _{p \rightarrow \infty}[p \tilde{K}(p)]=M(1-a) \geq 0$, the parameter $a$ satisfies the inequality $a \leq 1$. The phase speed is contained between $C_{\infty}>0$ and $C_{0}<\infty$, where $C_{0}:=c_{0}\left[1+K_{0} / \mathcal{K}\right]^{1 / 2}$ is the wavefront speed, the phase speed is bounded from below: $c(\omega) \geq c_{0}$, with $c_{0} \leq C_{0}$. The formula

$$
\tilde{f}(y)=y^{\alpha-1} /\left(1+y^{\alpha}\right)
$$

for the Laplace transform of $f(x)=\mathrm{E}_{\alpha}\left(-x^{\alpha}\right)$ yields the kernel $K$ :

$$
K(t)=M(1-a) \mathrm{E}_{\alpha}\left(-(t / \tau)^{\alpha}\right), \quad t \geq 0,
$$

where $\mathrm{E}_{\alpha}$ denotes the Mittag-Leffler function [53]. It is proved in Appendix B that $K(t)$ in equation 30 is CM. This function is shown in Fig. 2. The function has been calculated numerically using equation 39 . 


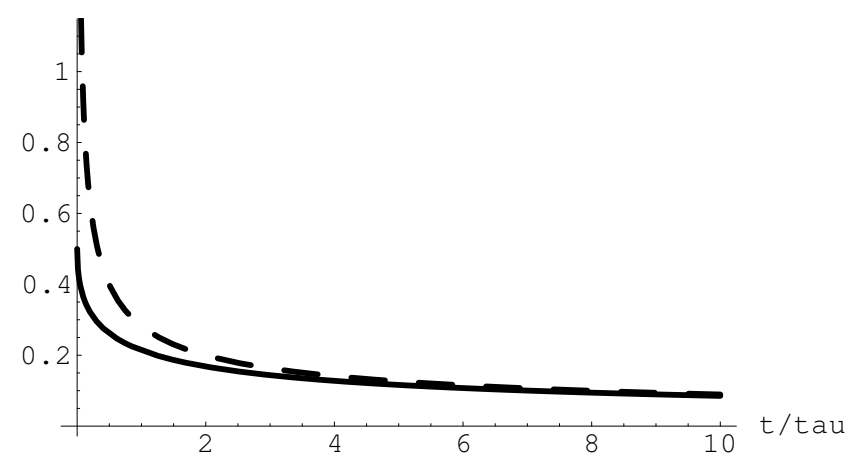

Figure 2: Comparison of the kernels $K(t)$ for the Cole-Cole model $(M / \mathcal{K}=1$, $a=0.5)$ and the constant- $Q$ model $(A / \mathcal{K}=0.5)$.

The high-frequency behavior of attenuation in the above model is given by the formula

$$
\mathcal{A}(\omega) \sim \frac{(1-a) M_{1} \sin (\alpha \pi / 2)}{2 c_{0} \tau\left(1+M_{1}\right)^{3 / 2}}(\tau \omega)^{1-\alpha},
$$

where $M_{1}=M / \mathcal{K}$. For $\omega \rightarrow 0$ we have a different picture:

$$
\mathcal{A}(\omega) \sim \frac{(1-a) M_{1} \sin (\alpha \pi / 2)}{2 c_{0} \tau\left(1+M_{1}\right)^{3 / 2}}(\tau \omega)^{1+\alpha}
$$

The exponent $1+\alpha$ lies between 1 and 2 , in accordance with experimental data for polymers and bio-tissues in the frequency range 0-250 $\mathrm{MHz}$.

The Cole-Cole attenuation function can be calculated numerically using the formula

$$
\begin{gathered}
\mathcal{A}(\omega)=\omega \sqrt{\sqrt{X(\omega)^{2}+Y(\omega)^{2}}-X(\omega)} /\left(2 c_{0} \sqrt{X(\omega)^{2}+Y(\omega)^{2}}\right) \\
X(\omega)=1+(M *(1-a) / \mathcal{K})(\omega \tau)^{\alpha}\left((\omega \tau)^{\alpha}+\cos (\pi \alpha / 2)\right) /\left(1+(\omega \tau)^{2 \alpha}+2\left(\omega \tau^{\alpha} \cos (\pi \alpha / 2)\right)\right. \\
Y(\omega)=-(M / \mathcal{K})(1-a)(\omega \tau)^{\alpha} \sin (\pi \alpha / 2) /\left(1+(\omega \tau)^{2 \alpha}+2(\omega \tau)^{\alpha} \cos (\pi \alpha / 2)\right)
\end{gathered}
$$

Using these formulas the functions $\mathcal{A}(\omega)$ and $c(\omega)$ were plotted in Fig. 3 for $c_{0}=$ $1500 \mathrm{~m} / \mathrm{s}, a=0.5, M / \mathcal{K}=1, K_{\infty} / \mathcal{K}=a, \tau=10^{-13} \mathrm{~s}$ and $\alpha=0.2,0.5$ and 0.8 . The plot shows that the Cole-Cole attenuation function obeys two approximate power laws, a superlinear one in the low frequency range $\omega \ll 3.27 \times 10^{6} \mathrm{MHz}$ and a sublinear one in the high frequency range $\omega \gg 3.27 \times 10^{6} \mathrm{MHz}$. 


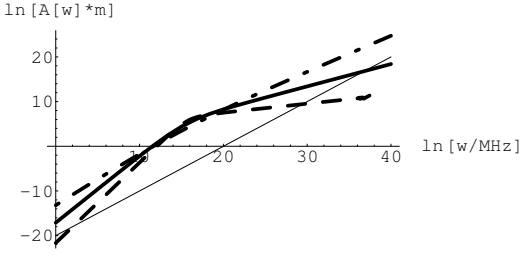

(a) Log-log plot of the attenuation function.

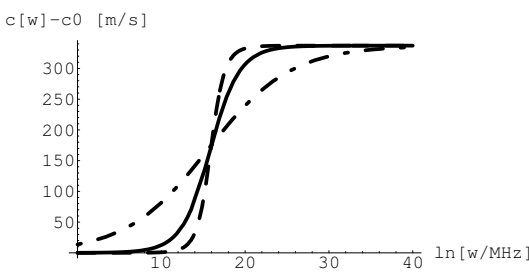

(b) Plot of phase speed vs $\ln (\omega / \mathrm{MHz})$.

Figure 3: Attenuation function and phase speed for the Cole-Cole relaxation model for $\alpha=0.2$ (dot-dash), 0.5 (solid), 0.8 (dash).

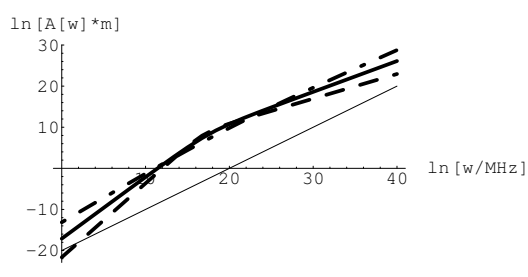

(a) Log-log plot of the attenuation function.

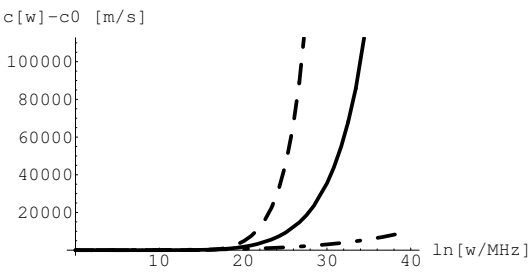

(b) Plot of phase speed vs $\ln (\omega / \mathrm{MHz})$.

Figure 4: Attenuation function and phase speed for the constant-Q model for $\alpha=0.2$ (dot-dash), 0.5 (solid), 0.8 (dash).

\subsection{A viscoelastic model with unbounded phase speed.}

If $K(t)=A(t / \tau)^{-\alpha} / \Gamma(1-\alpha)$ with $A, \tau>0$ and $0<\alpha<1$, then the pressure equation assumes the form

$$
c_{0}^{-2} P_{, t t}=\nabla^{2} P+\left(A \tau^{\alpha} / \rho_{0} c_{0}^{2}\right) \mathrm{D}^{\alpha} \nabla^{2} P
$$

where $\mathrm{D}^{\alpha}$ denotes the Caputo fractional time derivative of order $\alpha$. This particular equation was considered in [18]. It is related to the constant-Q model in seismology [54, 55]. In this case $K_{0}=\infty$, hence $C_{0}=\infty$ and the solutions do not exhibit wavefronts. In this case $X(\omega)=1+A_{1}(\tau \omega)^{\alpha} \cos (\pi \alpha / 2)$ and $Y(\omega)=A_{1}(\tau \omega)^{\alpha} \sin (\pi \alpha / 2)$, where $A_{1}:=A / \mathcal{K}$. The attenuation function and phase speed are shown for $A_{1}=0.5$ in Fig. 4, which confirms that the phase speed is unbounded. This is hardly surprising because the order of the derivatives in the last term of equation (34) is $2+\alpha$, higher than the orders of the other derivatives and the equation of motion is parabolic.

Asymptotic behavior of dispersion and attenuation associated with special wave equations based on fractional versions of spring-dashpot models has been examined in a series of papers by Holm, Näsholm and Sinkus [18, 24, 56, 57. The convolution kernels $K$ corresponding to the fractional spring-dashpot models are $\mathrm{CM}$, hence the general theory developed in Sec. 3.2 applies to their equations. 


\subsection{Quasi-linear attenuation.}

The attenuation function is almost linear while phase speed varies very slowly in seismological applications [26, 58, as well as in marine sediments 59]. This results in an approximately constant $Q$ factor, defined by the formula $Q(\omega):=$ $\omega /[2 c(\omega) \mathcal{A}(\omega)]$.

We shall use asymptotic considerations to investigate dispersion, attenuation and existence of wavefronts for a nearly linear attenuation function. Note that an exactly linear rate of growth of the attenuation function in the high frequency range would be inconsistent with the assumption that the origin of attenuation is purely viscoelastic and the relaxation modulus is CM. A model of a linear attenuation function and an approximately linear $\mathcal{D}(\omega)$ was elaborated by Futterman [26. Futterman was only concerned with finite phase speed for his strictly linear attenuation model. In contrast to Futterman we shall take into account the fact that attenuation grows at a strictly sublinear rate. Furthermore, the investigations of this section also shed some light on the relation between boundedness of the phase speed and existence of wavefronts. One might wonder whether it is possible that the phase speed has a finite upper bound $C_{0}$ but the wave field extends beyond the surface $|\mathbf{x}|=C_{0} t$. It will turn out that this can happen.

Note that the attenuation function in the constant- $Q$ model of the previous section is sublinear, while absence of wavefronts manifests itself through unboundedness of phase speed (Fig. 4). Sublinearity of the attenuation function alone does not however guarantee bounded phase speed. The Fourier transform of the one-dimensional Green's function is

$$
g(\omega)=F(\omega) \mathrm{e}^{-\mathrm{i} \omega\left(t-|\mathbf{x}| / C_{0}\right)-\mathrm{i} \mathcal{D}(\omega)|\mathbf{x}|-\mathcal{A}(\omega)|\mathbf{x}|}
$$

where $|F(\omega)|$ is bounded. The function $g$ is square integrable for $|\mathbf{x}|>0$ if $\mathcal{A}(\omega) /|\ln (\omega)|$ is unbounded for $\omega \rightarrow \infty$. Suppose that this condition is satisfied. According to the Paley-Wiener theorem (60, Theorem XII) Green's function vanishes for $t<|\mathbf{x}| / C_{0}$ if and only if

$$
\int_{0}^{\infty} \frac{\mathcal{A}(\omega)}{1+\omega^{2}} \mathrm{~d} \omega<\infty
$$

This is true in particular for

$$
\mathcal{A}(\omega) \sim a \omega^{1+\lambda} /|\ln (\omega)|^{\gamma}, \quad a>0, \quad \omega \rightarrow \infty
$$

if the following condition is satisfied:

(*) Either $\lambda<0$ or $\lambda=0$ and $\gamma>1+\varepsilon$.

In order to check the behavior of attenuation and phase speed in these cases we have to turn to equations $(19)$ and $(20)$. Consider a Radon measure $\nu(\mathrm{d} r)=h(r) \mathrm{d} r$, whose density has the asymptotic behavior $h(r) \sim b r^{\lambda} /|\ln r|^{\gamma}$ at infinity, where $b>0$. Inequality 18 is satisfied if

$$
b \int_{N}^{\infty} \frac{r^{\lambda} \mathrm{d} r}{(1+r) \ln (r)^{\gamma}} \equiv b \int_{\mathrm{e}^{N}}^{\infty} \frac{\mathrm{e}^{\lambda y} \mathrm{~d} y}{y^{\gamma}\left(1+\mathrm{e}^{-y}\right)}<\infty
$$


for some sufficiently large $N>1$, hence it is satisfied if and only if Condition $(*)$ is satisfied.

We shall now calculate the asymptotic behavior of the functions $\mathcal{A}(\omega)$ and $\mathcal{D}(\omega)$. In terms of the integration variable $s=r^{2}$

$$
\mathcal{A}(\omega)=\omega^{2} \int_{] 0, \infty[} \frac{\mu(\mathrm{d} s)}{\omega^{2}+s}
$$

where $\mu(\mathrm{d} s)=h(\sqrt{s}) \mathrm{d} s /(2 \sqrt{s}) \sim b s^{(\lambda-1) / 2} \mathrm{~d} s /\left(2|\ln (\sqrt{s})|^{\gamma}\right)$ for $s \rightarrow \infty$. Note that $1 /|\ln (\sqrt{s})|^{\gamma}$ is a slowly varying function. By Theorem 5

$$
\mathcal{A}(\omega) \sim(b / 2)[\pi / \cos (\pi \lambda / 2)] \omega^{1+\lambda} /|\ln (\omega)|^{\gamma}
$$

for $\omega \rightarrow \infty$. It is thus seen that in the case under consideration inequality 18 is equivalent to the statement that $|\mathbf{x}|=C_{0} t$ is a wavefront of Green's function and also to Condition $(*)$.

By a similar argument equation 20 implies that

$$
\mathcal{D}(\omega) \sim(b / 2)[\pi / \cos (\pi \lambda / 2)] \omega^{1+\lambda} /|\ln (\omega)|^{\gamma}
$$

and, using equation 21,

$$
c(\omega) \sim C_{0}\left[1-b \omega^{\lambda} /\left(2|\ln (\omega)|^{\gamma}\right]\right.
$$

If $\lambda<0$ or $\lambda=0$ and $\gamma>0$, then $\lim _{\omega \rightarrow \infty} c(\omega)=C_{0}$.

If $\lambda=0$ and $0<\gamma \leq 1$ then the phase speed has a finite upper bound $C_{0}$ but the Paley-Wiener theorem implies that the wavefield is not bounded by the surface $|\mathbf{x}|=C_{0} t$. It should however be noted that in this special case failure of inequality (18) implies that the kernel $K(t)$ cannot be locally integrable and CM. For $\lambda=0$ and $\gamma>0$ the dispersion is very weak. A nearly linear attenuation and a nearly constant phase speed is observed in marine sediments as well as in the Earth's crust and mantle.

\section{Conclusions.}

Consistency with linear viscoelasticity requires that attenuation in the pressure wave equation is represented by a term of the form $K * \nabla^{2} P$. The operator $\nabla^{2}$ is here crucial for truly viscoelastic attenuation.

If the kernel $K$ is completely monotonic then the wavenumber function $\kappa(p)$ is a $\mathrm{CBF}$ and a rich theory of dispersion-attenuation developed in 25, 17 applies. The general results presented in Sec. 3.2 has a very strong predictive power even before any particular model of the medium is substituted.

It was pointed out in Section 3.2 that $\mathcal{A}(\omega)$ is sublinear in the high-frequency range. This is also true when phase speed is unbounded and there are no wavefronts. As the results of Sections 3.2 and 4.4 indicate, absence of wavefronts manifests itself by unboundedness of the phase speed rather than by the Paley-Wiener criterion alone. On the other hand experimental data for many 
polymers, castor oil and bio-tissues indicate a power law for the attenuation function $\mathcal{A}(\omega)$ with an exponent in the range 1-2. Such values of the exponent in the high frequency range are incompatible with viscoelasticity but they are consistent with the low frequency asymptotics of the attenuation function. They are also incompatible with bounded phase speed. Viscoelasticity does not however exclude an unbounded phase speed. Comparison of characteristic relaxation times with the range of experimental data obtained for polymers and bio-tissues $(0-250 \mathrm{MHz})$ indicate that the observed data are pertinent for the low-frequency behavior of the attenuation function. Low-frequency behavior of attenuation and dispersion is examined in some more detail in the context of linear viscoelasticity in [17.

High-frequency asymptotic properties of the attenuation function are unavailable to direct measurements but they affect the singularity carried by the wavefront if $C_{0}<\infty$.

\section{References}

[1] T. L. Szabo. Time domain wave equations for lossy media obeying a frequency power law. J. Acoust. Soc. Am., 96:491-500, 1994.

[2] T. L. Szabo. Causal theories and data for acoustic attenuation obeying a frequency power law. J. Acoust. Soc. Am., 97:14-24, 1995.

[3] T. L. Szabo and J. Wu. A model for longitudinal and shear wave propagation in viscoelastic media. J. Acoust. Soc. Am., 107:2437-2446, 2000.

[4] T. L. Szabo. Diagnostic Ultrasound Imaging: Inside Out. Elsevier - Academic Press, Amsterdam, 2004.

[5] J. F. Kelly, R. J. McGough, and M. M. Meerschaert. Analytical timedomain Green's functions for power-law media. J. Acoust. Soc. Am., 124:2861-2872, 2008.

[6] P. Straka, M. M. Meerschaert, R. J. McGough, and Yuzhen Zhou. Fractional wave equations with attenuation. Fract. Calc. and Applications, 16:262-272, 2013.

[7] M. A. Biot. Theory of stress-strain relation in anisotropic viscoelasticity and relaxation phenomena. J. appl. Phys., 25:1385-1391, 1954.

[8] A. Hanyga and M. Seredyńska. Thermodynamics and asymptotic theory of wave propagation in viscoporous media. In Yu-Chiung Teng, Er-Chang Shang, Yih-Hsing Pao, M. H. Schultz, and A. D. Pierce, editors, Theoretical and Computational Acoustics '97, Singapore, 1999. World-Scientific. Proc. $3^{\text {rd }}$ Int. Conf. on Computational and Theoretical Acoustics, Newark, NJ, July 14-18, 1997. 
[9] A. A. Lokshin and V. E. Rok. Fundamental solutions of the wave equation with delayed time. Doklady AN SSSR, 239:1305-1308, 1978.

[10] A. Hanyga and V. E. Rok. Wave propagation in micro-heterogeneous porous media: a model based on an integro-differential equation. J. acoust. Soc. Am., 107:2965-2972, 2000.

[11] A. Hanyga and M. Seredyńska. Some effects of the memory kernel singularity on wave propagation and inversion in poroelastic media, I: Forward modeling. Geophys. J. Int., 137:319-335, 1999.

[12] Jian-Fei Lu and A. Hanyga. Wave field simulation for heterogeneous transversely isotropic porous media with the JKD dynamic permeability. Comp. Mech., 36:196-208, 2005.

[13] Jian-Fei Lu and A. Hanyga. Fundamental solution for a layered porous half-space subject to a vertical point force or a point fluid force. Comput. Mech., 35:376-391, 2005.

[14] A. N. Norris. On the viscodynamic operator in Biot's theory. J. WaveMaterial Interaction, 1:365-380, 1986.

[15] S. R. Pride, A. F. Gangi, and F. Dale Martin. Deriving the equations of porous saturated media. J. Acoust. Soc. Am., 92:3278-3290, 1992.

[16] S. R. Pride, J. G. Berryman, and J. M. Harris. Seismic attenuation due to wave-induced flow. J. Geophys. Res. B, 109:B01201, 2004.

[17] A. Hanyga. Wave propagation in linear viscoelastic media with completely monotonic relaxation moduli. Wave Motion, 50:909-928, 2013.

[18] S. Holm and R. Sinkus. A unifying fractional wave equation for compressional and shear waves. J. Acoust. Soc. Am., 127:542-548, 2010.

[19] R. Kowar, O. Scherzer, and Y. Bonnefond. Causality analysis of frequencydependent wave attenuation. Mathematical Methods in the Applied Sciences, 34:108-124, 2011.

[20] R. Kowar and O. Scherzer. Attenuation models in photoacoustics. In H. Ammari, editor, Mathematical Modeling in Biomedical Imaging II. Optical, Ultrasound, and Opto-Acoustic Tomographies, chapter 4, pages 85-130. Springer, Berlin, 2012.

[21] A. Hanyga. On wave propagation in viscoelastic media with concave creep compliance. arXiv:1302.1797 math-ph, to appear in Quart. J. Math. appl. Mech. 2013.

[22] A. J. Nachman, J. F. Smith III, and R. C. Waag. An equation for acoustic propagation in inhomogeneous media with relaxation losses. J. Acoust. Soc. Am., 88:1584-1595, 1990. 
[23] S. von Ende, A. Lion, and R. Lammering. On the thermodynamically consistent fractional wave equation for viscoelastic solids. Acta Mech., 221:1$10,2011$.

[24] S. P. Näsholm and S. Holm. Linking multiple relaxation, power-law attenuation and and fractional wave equations. J. Acoust. Soc. Am., 130:30383045, 2011.

[25] A. Hanyga and M. Seredyńska. Spatially fractional-order viscoelasticity, non-locality, and a new kind of anisotropy. Journal of Mathematical Physics, 53:052902, 2012.

[26] W. Futterman. Dispersive body waves. J. Geophys. Res., 67:5279-5291, 1962.

[27] M. J. Buckingham. Theory of acoustic attenuation, dispersion and pulse propagation in unconsolidated granulated materials including marine sediments. J. Acoust. Soc. Am., 102:2579-2596, 1997.

[28] Y.-M. Jiang. Measurement of low-frequency sound attenuation in marine sediment. IEEE Journal of Oceanic Engineering, 35:70-78, 2010.

[29] A. Hanyga and M. Seredyńska. Relations between relaxation modulus and creep compliance in anisotropic linear viscoelasticity. J. of Elasticity, 88:41$61,2007$.

[30] A. N. Beris and B. J. Edwards. On the admissibility criteria for linear viscoelastic kernels. Rheologica Acta, 32:505-510, 1993.

[31] W. A. Day. Restrictions on the relaxation functions in linear viscoelasticity. Quart. Jl Mech. appl. Math., 24:487-497, 1970.

[32] R. S. Anderssen and R. J. Loy. Completely monotone fading memory relaxation moduli. Bull. Austr. Math. Soc., 65:449-460, 2002.

[33] R. L. Schilling, R. Song, and Z. Vondraček. Bernstein Functions. Theory and Applications. De Gruyter, Berlin, 2010.

[34] G. Gripenberg, S. O. Londen, and O. J. Staffans. Volterra Integral and Functional Equations. Cambridge University Press, Cambridge, 1990.

[35] D. F. Shea. One complement to Valiron's Tauberian theorem for the Stieltjes transform. Proc. Amer. Math. Soc., 21:1-9, 1969.

[36] K. F. Herzfeld and A. Litovitz. Absorption and Dispersion of Ultrasonic Waves. Academic Press, New York, 1959.

[37] S. I. Rokhlin, D. K. Lewis, K. F. Graff, and L. Adler. Real-time study of frequency dependence of attenuation and velocity of ultrasonic waves during the curing reaction of epoxy resin. J. Acoust. Soc. Am., 79:1786$1793,1986$. 
[38] H. M. Nussenzveig. Causality and Dispersion Relations. Academic Press, New York, 1972.

[39] M. Renardy. Some remarks on the propagation and non-propagation of discontinuities in linearly viscoelastic liquids. Rheol. Acta, 21:251-254, 1982.

[40] W. Hrusa and M. Renardy. On wave propagation in linear viscoelasticity. Quart. appl. Math., 43:237-253, 1985.

[41] W. Hrusa and M. Renardy. On a class of quasilinear partial integrodifferential equations with singular kernels. J. Diff. Eqs, 64:195-220, 1986.

[42] W. Hrusa and M. Renardy. A model equation for viscoelasticity with a strongly singular kernel. SIAM J. Math. Anal., 19:257-269, 1988.

[43] W. Desch and R. Grimmer. Propagation of singularities for integrodifferential equations. J. Diff. Eqs, 65:411-426, 1965.

[44] W. Desch and R. Grimmer. Singular relaxation moduli and smoothing in three-dimensional viscoelasticity. Trans. Amer. Math. Soc., 314:381-404, 1989 .

[45] A. Hanyga and M. Seredyńska. Asymptotic ray theory in poro- and viscoelastic media. Wave Motion, 30:175-195, 1999.

[46] A. Hanyga and M. Seredyńska. Asymptotic wavefront expansions in hereditary media with singular memory kernels. Quart. Appl. Math., LX:213-244, 2002.

[47] E. Strick. Application of linear viscoelasticity to seismic wave propagation. In F. Mainardi, editor, Wave Propagation in Viscoelastic Media, London, 1982. Pitman.

[48] E. Strick. A predicted pedestal effect for a pulse propagating in constant Q solids. Geophysics, 35:387-403, 1970.

[49] M. D. Verweij. Modeling space-time domain acoustic wave fields in media with attenuation: The symbolic manipulation approach. J. Acoust. Soc. Am., 97:831-843, 1994.

[50] H.-P. Liu, D. L. Anderson, and H. Kanamori. Velocity dispersion due to anelasticity; implications for seismology and mantle composition. Geophys. J. R. astr. Soc., 47:41-58, 1976.

[51] K. S. Cole and R. H. Cole. Dispersion and absorption in dielectrics, I: Alternating current characteristics. J. Chem. Phys., 9:341-351, 1941.

[52] R. L. Bagley and P. J. Torvik. On the fractional calculus model of viscoelastic behavior. J. of Rheology, 30:133-155, 1986. 
[53] I. Podlubny. Fractional Differential Equations. Academic Press, San Diego, 1998.

[54] E. Kjartansson. Constant Q-wave propagation and attenuation. J. Geophys. Res., 84:4737-4748, 1979.

[55] J. M. Carcione, F. Cavallini, F. Mainardi, and A. Hanyga. Time-domain seismic modeling of constant-Q wave propagation using fractional derivatives. Pure appl. Geophys., 159:1714-1736, 2002.

[56] S. P. Näsholm and S. Holm. A fractional acoustic equation from multiple relaxation loss and conservation laws. arXiv:1202.4251v1, 2012. Presented at the 5th IFAC Symposium on Fractional Differentiation and Its Applications (FDA 2012), Hohai University, Nanjing, China, 14-17 May 2012.

[57] S. P. Näsholm and S. Holm. On a fractional Zener elastic wave equation. Fract. Calc. Appl. Anal., 16:26-50, 2013.

[58] E. Strick. The determination of Q, dynamic viscosity and creep curves from wave propagation measurements. Geophys. J. Roy. astr. Soc., 13:197-218, 1967.

[59] M. J. Buckingham. On pore-fluid viscosity and the wave properties of saturated granular materials including marine sediments. J. Acoust. Soc. Am., 122:14861501, 2007.

[60] R. E. A. C. Paley and N. Wiener. Fourier Transforms in the Complex Domain. AMS, New York, 1934.

[61] A. Hanyga. Multi-dimensional solutions of time-fractional diffusion-wave equations. Proc. Roy. Soc. London A, 458:933-958, 2002.

\section{A Solution of the Cauchy problem $7-9$}

Consider the initial value problem defined by equation 77 in one-dimensional space with the initial conditions $P(0, x)=P_{0}(x)$ and $P_{, t}(0, x)=Q_{0}(x)$. The Laplace-Fourier transform

$$
\hat{\tilde{P}}(p, k):=\int_{0}^{\infty} \mathrm{e}^{-p t}\left[\int_{-\infty}^{\infty} \mathrm{e}^{-\mathrm{i} k x} P(t, x) \mathrm{d} x\right] \mathrm{d} t
$$

is given by the expression $\hat{\tilde{P}}(p, k)=F(p, k) /\left[k^{2}+\kappa(p)^{2}\right]$, where

$$
F(p, k):=c_{0}^{-2} \frac{\left[p\left(1+k^{2} / \rho\right) \hat{P}_{0}(k)+\hat{Q}_{0}(k)\right]}{1+\left(\rho_{0} c_{0}^{2}\right)^{-1} p \tilde{K}(p)}
$$

Hence

$$
P(t, x)=\frac{1}{2 \pi \mathrm{i}} \int_{-\mathrm{i} \infty+\varepsilon}^{\mathrm{i} \infty+\varepsilon} \mathrm{e}^{p t}\left[\frac{1}{2 \pi} \int_{-\infty}^{\infty} \mathrm{e}^{\mathrm{i} k x} F(p, k) \frac{1}{k^{2}+\kappa(p)^{2}} \mathrm{~d} k\right] \mathrm{d} p
$$


for an arbitrary $\varepsilon>0$.

If $x>0 / x<0$ then the contour of the inner integral can be closed by a semicircle at infinity in the upper/lower complex $k$-half-plane, $\operatorname{Im} k>0 / \operatorname{Im} k<$ 0 . Since $\operatorname{Re} \kappa(-\mathrm{i} \omega)=\mathcal{A}(\omega) \geq 0$, the only residue in the upper/lower $k$-halfplane is $k=\mathrm{i} \kappa(-\mathrm{i} \omega) / k=-\mathrm{i} \kappa(-\mathrm{i} \omega)$, hence

$P(t, x)=\frac{1}{4 \pi \mathrm{i}} \int_{-\mathrm{i} \infty+\varepsilon}^{\mathrm{i} \infty+\varepsilon} F_{1}(p) \mathrm{e}^{p t-\kappa(p)|x|} \mathrm{d} p=\frac{1}{4 \pi \mathrm{i}} \int_{-\mathrm{i} \infty+\varepsilon}^{\mathrm{i} \infty+\varepsilon} F_{1}(p) \mathrm{e}^{p(t-B|x|)-\beta(p)|x|} \mathrm{d} p$

where $F_{1}(p):=F(p, \kappa(p) \operatorname{sgn} x) / \kappa(p)$.

For $t<B|x|$ the Bromwich contour can be closed in the right $p$-half-plane Re $p>0$. The function $\kappa(p)$ does not have any singularity except for a cut along the negative $p$-semi-axis. The integrand does not have any singularity in the right-half plane, hence $P(t, x)=0$ for $t<B|x|$. If $B>0$ then $C_{0}:=1 / B$ can be identified as the wavefront speed. If $B=0$ then $F(t, x)$ vanishes for $t<0$, which implies causality.

Define the function $Q(t, y)$ in such a way that $P(t, x)=Q(t,|x|)$. The solution $P^{(3)}(t, \mathbf{x})$ of the same initial-value problem in three dimensions is then given by the formula 61 .

$$
P^{(3)}(t, \mathbf{x})=-\frac{1}{2 \pi r} \frac{\partial Q(t, r)}{\partial r}
$$

where $r=|\mathbf{x}|$, and therefore $P^{(3)}(t, \mathbf{x})$ vanishes for $t<B r$.

\section{B The Cole-Cole relaxation kernel.}

The Mittag-Leffler function $\mathrm{E}_{\alpha}\left(-x^{\alpha}\right), 0<\alpha \leq 1$, can be calculated by applying the inverse Laplace transform to equation (29):

$$
\mathrm{E}_{\alpha}\left(-x^{\alpha}\right)=\frac{1}{2 \pi \mathrm{i}} \int_{\varepsilon-\mathrm{i} \infty}^{\varepsilon+\mathrm{i} \infty} \mathrm{e}^{y x} \frac{y^{\alpha-1}}{y^{\alpha}+1} \mathrm{~d} y
$$

where $\varepsilon>0$. The Bromwich contour can be deformed to a Hankel loop running along a the straight line $\mathcal{L}_{-}$from $-\infty-\mathrm{i} \varepsilon$ to $-\mathrm{i} \varepsilon$, then along a semicircle of radius $\varepsilon$ centered at 0 and along the straight line $\mathcal{L}_{+}$from $\mathrm{i} \varepsilon$ to $-\infty+\mathrm{i} \varepsilon$. The integral over the semi-circle

$$
\int_{-\pi}^{\pi} \mathrm{e}^{\varepsilon x \exp (\mathrm{i} \varphi)} \frac{\varepsilon^{\alpha-1} \exp (\mathrm{i}(\alpha-1) \varphi)}{1+\varepsilon^{\alpha} \exp (\mathrm{i} \alpha \varphi)} \mathrm{i} \varepsilon \mathrm{e}^{\mathrm{i} \varphi} \mathrm{d} \varphi
$$

tends to 0 as $\varepsilon \rightarrow 0$. The remaining two integrals tend to

$$
\frac{1}{\pi} \int_{0}^{\infty} \mathrm{e}^{-r t} \operatorname{Im}\left[\frac{r^{\alpha-1} \exp (-\mathrm{i}(\alpha-1) \pi)}{1+r^{\alpha} \exp (-\mathrm{i} \alpha \pi)}\right] \mathrm{d} r
$$


where $r \exp ( \pm \mathrm{i} \pi)$ has been substituted for $y$ on $\mathcal{L}_{ \pm}$. Equation 38 works out to

$$
\mathrm{E}_{\alpha}\left(-x^{\alpha}\right)=\frac{\sin (\alpha \pi)}{\pi} \int_{0}^{\infty} \mathrm{e}^{-r x} r^{\alpha-1}\left[r^{2 \alpha}+2 r^{\alpha} \cos (\alpha \pi)+1\right]^{-1} \mathrm{~d} r
$$

Equation (38) shows that $\mathrm{E}_{\alpha}\left(-x^{\alpha}\right)$ is the Laplace transform of a nonnegative function. By Theorem 1 it is CM.

The Cole-Cole kernel function 30 differs from $\mathrm{E}_{\alpha}\left(-x^{\alpha}\right)$ by a linear scaling transformation, hence it is CM. 